01

\title{
Моделирование распространения электромагнитных волн по поверхности человеческого тела
}

\author{
(С) И.Б. Вендик, О.Г. Вендик, В.В. Кириллов, В.В. Плескачев, П.А. Туральчук \\ Санкт-Петербургский государственный электротехнический университет „ЛЭТИ“, \\ 197376 Санкт-Петербург, Россия \\ e-mail: ibvendik@rambler.ru
}

(Поступило в Редакцию 12 января 2016 г.)

\begin{abstract}
Приведены результаты моделирования и экспериментального исследования электромагнитных (ЭМ) волн микроволнового диапазона, распространяющихся по поверхности человеческого тела. Исследованы также параметры распространения волны, как затухание и фазовая скорость. Выполнен расчет распространения ЭМ волн численным методом FDTD (метод конечных разностей во временно́й области), а также с использованием аналитической модели распространения ЭМ волны вдоль плоской и криволинейной поверхностей. Экспериментальное исследование проведено на живом объекте. Показано, что ползущие волны испытывают заметную дисперсию и являются замедленными, в то время как поверхностные волны дисперсии не имеют и распространяются со скоростью света в свободном пространстве. Проведено сравнение результатов численного моделирования, аналитического расчета и экспериментальных исследований на частоте $2.55 \mathrm{GHz}$
\end{abstract}

\section{Введение}

Моделирование распространения электромагнитных (ЭМ) волн по поверхности человеческого тела предполагает использование методики расчета электромагнитных структур, учитывающей особенности такого объекта. Существует два класса методов расчета ЭМ структур — численные и аналитические, позволяющие рассчитывать распределение ЭМ полей, используя известные методы решения уравнений Максвелла для выбранных граничных условий.

Численные методы, используемые в современных САПР, позволяют рассчитывать распространение электромагнитных волн по поверхности практически любых объектов. Специфической особенностью объекта исследования - человеческого тела — является разнообразие размеров и параметров, что требует проведения отдельного расчета для каждого конкретного случая. Использование упрощенных аналитических моделей для исследования распространения ЭМ волн вдоль поверхности человеческого тела позволяет получить приближенное решение при достаточно малых затратах вычислительных ресурсов и времени для каждого конкретного случая. При всем разнообразии форм поверхности человеческого тела можно выделить два основных вида: плоские и криволинейные поверхности. В действительности плоские поверхности имеют некоторую кривизну, а сечения округлых поверхностей не могут быть в точности описаны кривыми второго порядка, однако с точки зрения практических расчетов этими неидеальностями реальных форм человеческого тела можно пренебречь. Далее будем рассматривать распространение ЭМ волны только вдоль указанных типов поверхностей: плоской и криволинейной, соответствующей поверхности цилиндра круглого сечения (т.е. поверхности второго порядка).

\section{1. Типы волн, распространяющихся по поверхности человеческого тела}

На границе поверхности раздела воздух-среда могут возникать следующие типы волн $[1,2]$ :

- вытекающие волны (Leaky waves, LW), затухающие при распространении нормально к поверхности; излучаются преимущественно под некоторым углом к диэлектрической поверхности, зависящим от диэлектрической проницаемости материала диэлектрика;

- поверхностные волны (Surface waves, SW), pacпространяющиеся вдоль плоской поверхности, быстро затухающие при удалении от поверхности;

- ползущие волны (Creeping waves, CW), pacпространяющиеся вдоль криволинейной поверхности по касательной, способные огибать поверхность, попадая в область тени.

На рис. 1 схематически представлены волны различных типов, существующие на поверхности сложной формы.

Следует отметить, что возникновение тех или иных типов волн, распространяющихся вдоль поверхности границы раздела, зависит от свойств границы и частоты падающей на границу ЭМ волны [2,3]. В [3-5] рассматривается два варианта: плоская задача и случай криволинейной поверхности границы раздела. Исследуются условия распространения волн в таких структурах. Для плоского случая в низкочастотной области существуют затухающие вытекающие волны, а начиная с некоторой частоты, основными распространяющимися волнами являются поверхностные. Для криволинейной поверхности возможно распространение волн трех типов: вытекающие, поверхностные и ползущие. Концепция ползущих волн на криволинейной поверхности, соответствующей границе раздела двух сред с разными диэлектрическими 


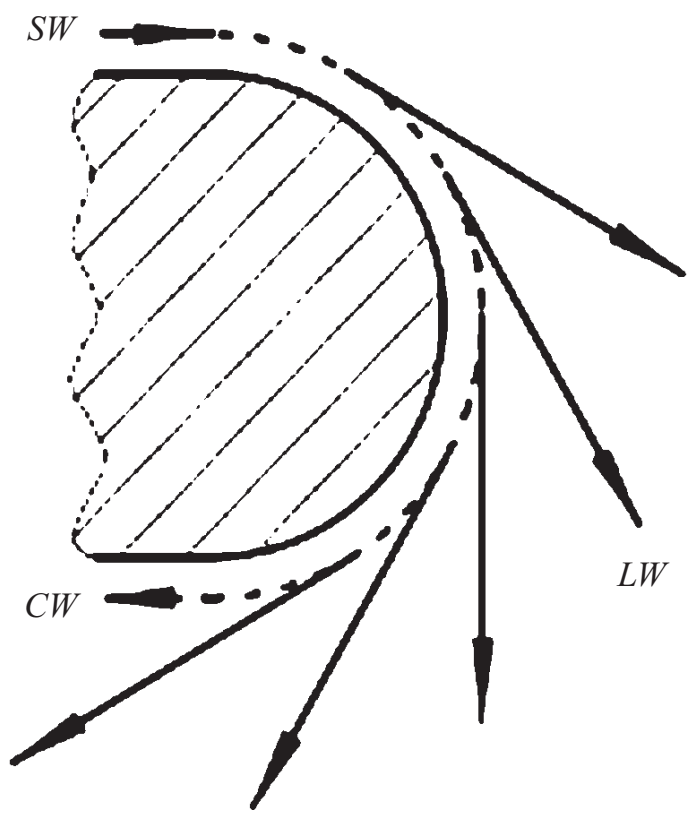

Pис. 1. Типы волн на поверхности сложной формы. свойствами, предложена и анализировалась в [6-9]. Для выбранной частоты тип распространяющейся волны зависит от геометрических размеров (радиуса цилиндра, толщины диэлектрического покрытия) и диэлектрической проницаемости диэлектрической оболочки цилиндра. ЭМ волна может распространяться не только по поверхности тела человека, но и проходить сквозь него, образуя дополнительный путь между источником и приемником излучения. Однако расчеты, представленные в $[10,11]$, показывают, что человеческое тело хорошо экранирует такое распространение в микроволновом диапазоне частот, делая вклад этой компоненты пренебрежимо малым по сравнению с энергией, переносимой волнами, распространяющимися по поверхности тела.

\section{2. Расчет распространения ЭМ волн методом FDTD}

Для расчета параметров ЭМ волн, распространяющихся по поверхности человеческого тела, в насто- $a$
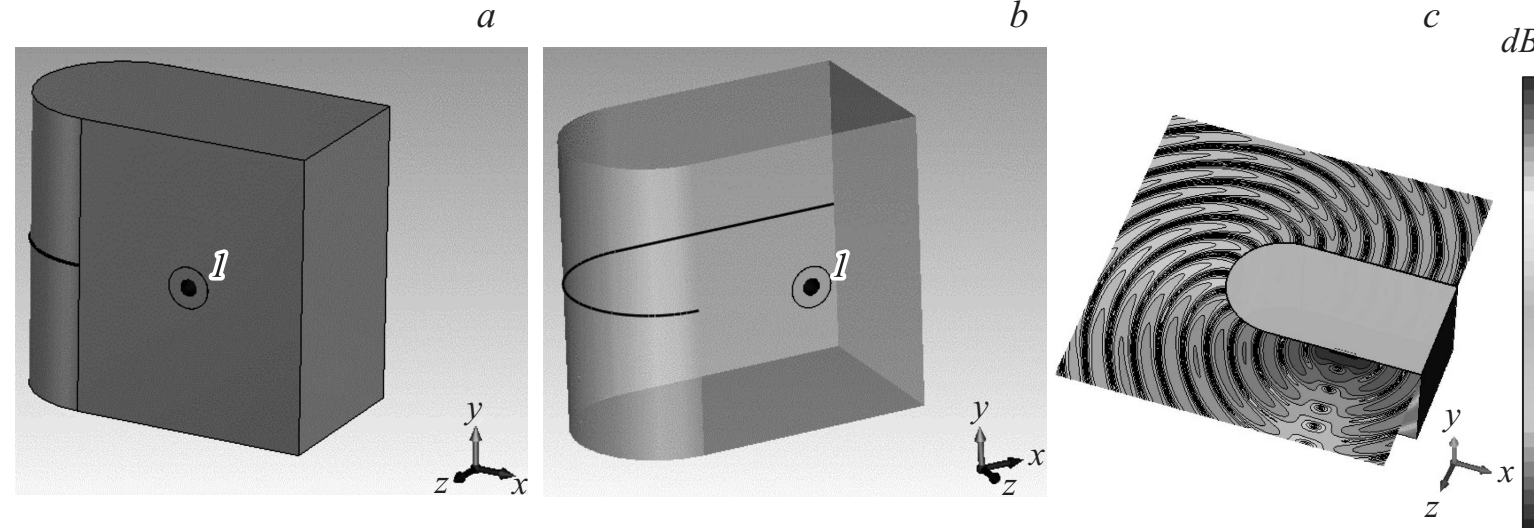

$d B, 1 \mathrm{~V} / \mathrm{m}$

40

29.1

18.2

7.27

$-3.64$

$-14.5$

$-25.5$

$-36.4$

$-47.3$

$-58.2$

$-69.1$
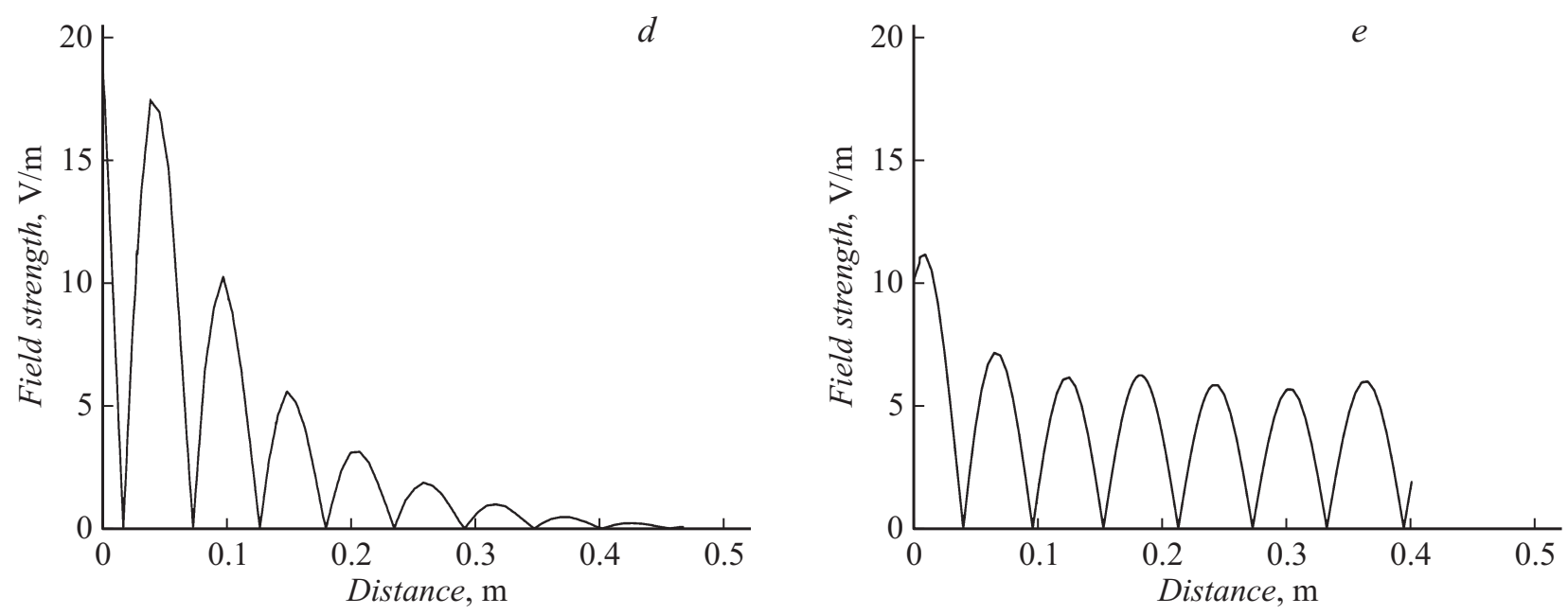

Рис. 2. Фантом человеческого тела для моделирования распространения поверхностных ЭМ волн численным методом - вид спереди $(a)$, вид с наложением пути расчета полей $(b)$. Результаты электродинамического моделирования на частоте $2.45 \mathrm{GHz}$ распределение модуля напряженности электрического поля ЭМ волны в поперечном сечении фантома $(c)$, на поверхности полуцилиндра $(d)$, на задней плоской поверхности $(e)$. 

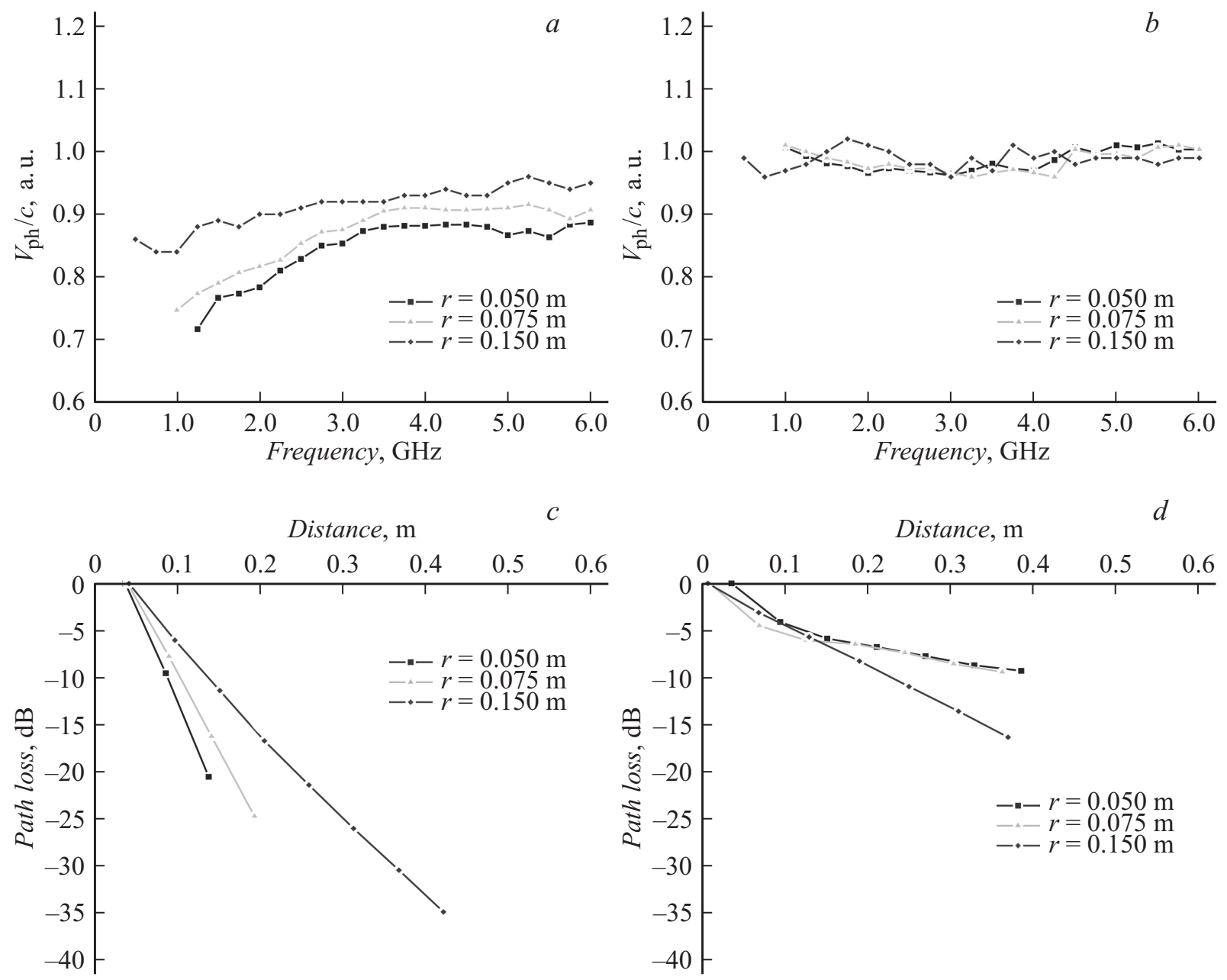

Рис. 3. Численный расчет зависимости нормированной фазовой скорости ползущей $(a)$ и поверхностной $(b)$ волн от частоты для фантома из идеального проводника. Потери распространения ползущей $(c)$ и поверхностной $(d)$ волн от расстояния для различных радиусов цилиндрической поверхности фантома из идеального проводника.

ящей работе применялся метод конечных разностей во временной области (FDTD). Используем упрощенную форму геометрической модели человеческого тела (фантома): прямоугольный параллелепипед и цилиндр той же высоты, соединенный с параллелепипедом (рис. 2,a). Относительная диэлектрическая проницаемость тканей человеческого тела в микроволновом диапазоне достаточно высокая $\left(\varepsilon_{r}=40-50\right)$ [6], что позволяет в оценочных расчетах параметров ЭМ волны заменить материал фантома на идеальный проводник. Толщина параллелепипеда совпадает с диаметром цилиндра $r=0.15 \mathrm{~m}$ (рис. $2, a-c$ ). Плоские вертикальные поверхности цилиндра имитируют грудную клетку и спину человека, а боковая поверхность полуцилиндра соответствует области прижатых рук и плеч. Дисковая антенна, возбуждающая поверхностные ЭМ волны, расположена в центре поверхности „груди“ фантома, как показано на рис. $2, a$. Расчет модуля напряженности ЭМ поля производится вдоль кривой, изображенной на рис. $2, b$.
Произведем оценку длины ЭМ волны, распространяющейся по поверхности человеческого тела. Длина волны оценивается по расстоянию между соседними минимумами электрического поля, рассчитанного на поверхности фантома вдоль выбранной траектории. Длина волны $\lambda$ рассчитывается как удвоенное расстояние $l$ между двумя соседними минимумами модуля напряженности электрического поля ЭМ волны

$$
\lambda=2 l \text {. }
$$

Траектория включает в себя как плоский участок (поверхность „спины“), так и участок в виде окружности („плечо“), как показано на рис. $2, b$, что позволяет найти длину поверхностной и ползущей волн соответственно. В качестве примера на рис. 2, с представлено распределение модуля напряженности электрического поля ЭМ волны на частоте $2.45 \mathrm{GHz}$, полученное в результате численного электродинамического моделирования. На рис. $2, d$ и $e$ приведено распределение модуля напряженности поля по боковой и по плоской 
поверхностям фантома соответственно. Точка начала отсчета, т.е. нуль по оси абсцисс на графике рис. 2, $d$, соответствует точке поперечного сечения, в которой передняя поверхность фантома с излучающей антенной соприкасается с боковой криволинейной поверхностью, а точка начала отсчета на графике рис. 2, е соответствует точке поперечного сечения, в которой боковая поверхность касается задней поверхности фантома.

Анализ зависимостей распределения поля от расстояния, представленных на рис. $2, d, e$, подтверждает, что по плоской поверхности фантома распространяется поверхностная волна, потери которой относительно медленно увеличиваются с расстоянием, а по боковой криволинейной поверхности распространяется ползущая волна, потери которой связаны с частичным переизлучением энергии в пространство по мере ее распространения (вытекающие волны). Также на указанных рисунках четко видны минимумы модуля вектора напряженности электрического поля, расстояние между которыми позволяет рассчитать длину волны по формуле (1).

Исследуем зависимость фазовой скорости поверхностной и ползущей ЭМ волн от частоты $f$. Зная длину волны $\lambda$ и частоту ЭМ волны $f$, рассчитаем фазовую скорость $v_{p h}$, используя известное соотношение

$$
v_{p h}=f \lambda
$$

На рис. 3, $a, b$ представлены результаты расчета частотной зависимости фазовой скорости ЭМ волны, распространяющейся по боковой (криволинейной) и задней (плоской) поверхностям фантома из идеального проводника. Ошибка расчета фазовой скорости по зависимости модуля напряженности электрической компоненты ЭМ волны вдоль боковой и задней поверхностей фантома от смещения по отношению к точке отсчета не более $\pm 5 \%$. Результаты расчета показывают, что дисперсия поверхностной ЭМ волны практически отсутствует. Ползущая волна обладает дисперсией, что проявляется в уменьшении фазовой скорости волны по мере уменьшения частоты. С увеличением частоты фазовая скорость этой волны асимптотически стремится к скорости света в вакууме. Эффект асимптотического увеличения скорости ползущей волны с частотой можно объяснить уменьшением длины волны по сравнению с радиусом поверхности, т.е. с ростом частоты криволинейная поверхность по отношению к распространяющейся ЭМ волне становится все более „Плоской“.

Расчет потерь выполнен на частоте $2.45 \mathrm{GHz}$ по результатам распределения модуля напряженности электрической компоненты ЭМ волны вдоль боковой и плоской (задней) поверхностей фантома (рис. $3, c-d)$. Расчеты проводились как для фантома из идеального диэлектрика, так и для фантома с потерями $(\varepsilon=42.5$ и $\sigma=1.66 \mathrm{~S} / \mathrm{m})$. Нормировка выполнялась по отношению к максимальной величине напряженности электрического поля (величина наибольшего из пиков распределения). Просачивание ЭМ энергии через объем диэлектрического фантома с потерями приводит к некоторому увеличению потерь. Зависимости потерь распространения поверхностной ЭМ волны вдоль плоской поверхности для фантомов различных размеров мало отличаются друг от друга. Изменение радиуса боковой цилиндрической поверхности фантома существенным образом влияет как на фазовую скорость ползущей ЭМ волны, так и на ее потери.

\section{3. Аналитическая модель распространения ЭМ волны вдоль плоской и криволинейной поверхностей}

Аналитический расчет параметров распространения ЭМ волн над плоской и криволинейной диэлектрическими поверхностями с потерями был предметом интенсивных исследований первой половины XX века в связи с развитием радиосвязи на длинных и коротких волнах. Большинство исследований относится к построению модели распространения ЭМ волны вдоль поверхности Земли. Поверхность Земли в простейшем случае можно представить как плоскую для связи на коротких расстояниях или круглую для дальней связи. Фундаментальные работы [12-14] рассматривают распространение ЭМ волн над плоской и круглой земной поверхностью соответственно.

Исследуем распространение ЭМ волны по плоской поверхности с потерями, воспользовавшись методом, предложенным в работах [12-14]. На рис. 4, $a$ изображена геометрическая схема распространения ЭМ волны над плоской диэлектрической поверхностью.

Одиночный излучатель $I d l$ расположен на расстоянии $a$ над диэлектрической поверхностью. Точка наблюдения удалена от центра координат на расстояние $r$ и находится на расстоянии $z$ от поверхности. ЭМ волна включает три компоненты: падающая, отраженная и распространяющаяся вдоль поверхности. Сумма этих компонент представлена следующим выражением для напряженности электрического поля $E_{z}$ :

$$
\begin{aligned}
E_{z} & =-\frac{j k_{0} I d l Z_{0} r^{2}}{4 \pi}\left[\frac{e^{-j k_{0} R_{2}}}{R_{1}^{3}}+\Gamma_{v} \frac{e^{-j k_{0} R_{2}}}{R_{2}^{3}}\right. \\
& \left.+\left(1-\Gamma_{v}\right)\left(1-j(\pi w)^{1 / 2}\right) e^{-e} \operatorname{erfc}\left(j w^{1 / 2}\right) \frac{e^{-j k_{0} R_{2}}}{R_{2}^{3}}\right] .
\end{aligned}
$$

В выражении (3) наименования геометрических параметров соответствуют рис. 4, $a$. Остальные параметры имеют следующий смысл: волновое число

$$
k_{0}=\frac{2 \pi}{\lambda}
$$



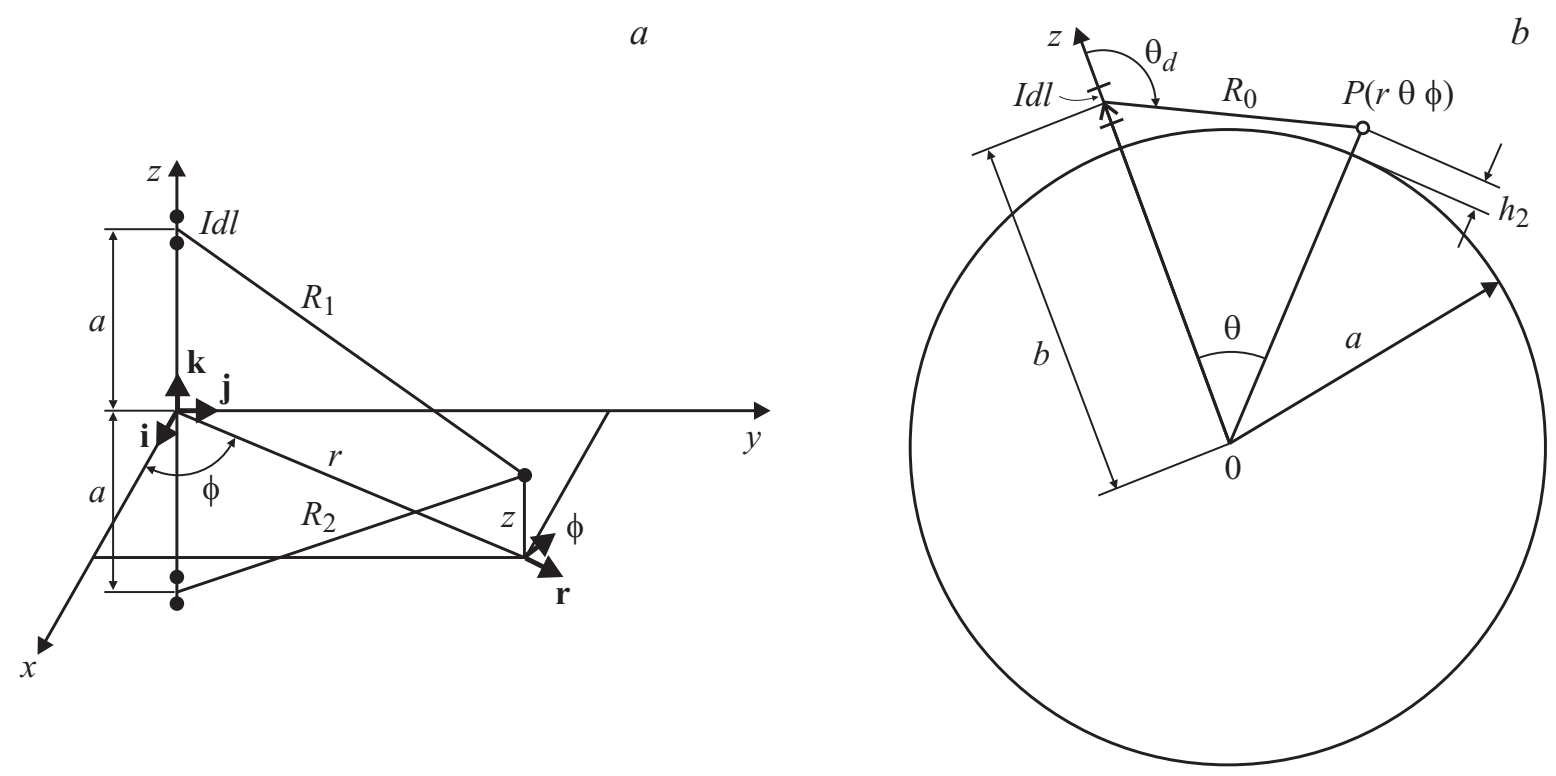

Рис. 4. Геометрическая схема распространения ЭМ волн над плоской $(a)$ и цилиндрической $(b)$ поверхностями (оригинальные иллюстрации из $[11,12])$.

зависящее от длины волны в свободном пространстве $\lambda$, волновое сопротивление свободного пространства:

$$
Z_{0}=\left(\frac{\mu_{0}}{\varepsilon_{0}}\right)^{1 / 2}=120 \pi
$$

волновое число $k_{1}$ в диэлектрической среде с относительной диэлектрической проницаемостью $\varepsilon_{r}$ и удельной проводимостью $\sigma$

$$
k_{1}=k_{0}\left[\varepsilon_{r}-j \frac{Z_{0}}{2 \pi} \sigma \lambda_{0}\right]^{1 / 2}
$$

и нормированный импеданс диэлектрической среды $\Delta_{0}$, определяемый отношением волнового сопротивления среды с учетом потерь к волновому сопротивлению свободного пространства:

$$
\Delta_{0}=\frac{k_{0}}{k_{1}}\left[1-j\left(\frac{k_{0}}{k_{1}} \frac{r}{R_{2}}\right)^{2}\right]^{1 / 2},
$$

параметры $\Gamma_{v}$ и $w$ определяются следующими выражениями:

$$
\begin{gathered}
\Gamma_{v}=\frac{a+z-\Delta_{0} R_{2}}{a+z+\Delta_{0} R_{2}}, \\
w=-\frac{j k_{0} R_{1}^{3}}{2 r^{2} R_{2}^{2}}\left(a+z+\Delta_{0} R_{2}\right)^{2} .
\end{gathered}
$$

Процесс распространения ползущей волны по поверхности окружности принципиальным образом отличается от процесса распространения волны по плоской поверхности. Выражения для расчета потерь распространения ползущей волны в наиболее удобном для аналитических расчетов виде приведены в [15]. На рис. 4, $b$ показана геометрия структуры, в которой распространяется ползущая волна. Так же, как и при распространении плоской волны, представим излучающий элемент в виде электрического диполя $I d l$, расположенного на расстоянии $b$ от центра вдоль радиуса окружности.

Для расчета затухания необходимо определить радиальную компоненту электрического поля на расстоянии $r$ от центра цилиндра в направлении радиуса, зависящую от угла $\Theta$ :

$$
\begin{aligned}
E_{r}= & -2 \frac{j k_{0} a I d l Z_{0}}{4 \pi b} \frac{e^{-j k_{0} a \Theta}}{e} e^{-j \frac{\pi}{4}}(\pi C \Theta)^{1 / 2} \\
& \times \sum_{s=1}^{\infty} \frac{e^{-j C \Theta t_{s}}}{t_{s}-q^{2}} \frac{w_{1}\left(t_{s}-\frac{k_{0}(b-a)}{C}\right) w_{1}\left(t_{s}-\frac{k_{0}(r-a)}{C}\right)}{w_{1}\left(t_{s}\right) w_{1}\left(t_{s}\right)}
\end{aligned}
$$

Приведенное выражение в отличие от (3) не содержит отдельных компонент, соответствующих падающей и отраженной волнам, так как уравнение является решением общего дифференциального уравнения:

$$
\frac{d w_{1}(t)}{d t}-q w_{1}(t)=0
$$

в котором $w_{1}(t)-$ функция Эйри-Фока [7],

$$
\begin{gathered}
q=-j C \Delta_{0}, \\
C=\left(\frac{k_{0} a}{2}\right)^{1 / 3}, \\
\Delta_{0}=\frac{k_{0}}{k_{1}}\left[1-j\left(\frac{k_{0}}{k_{1}}\right)^{2}\right]^{1 / 2} .
\end{gathered}
$$




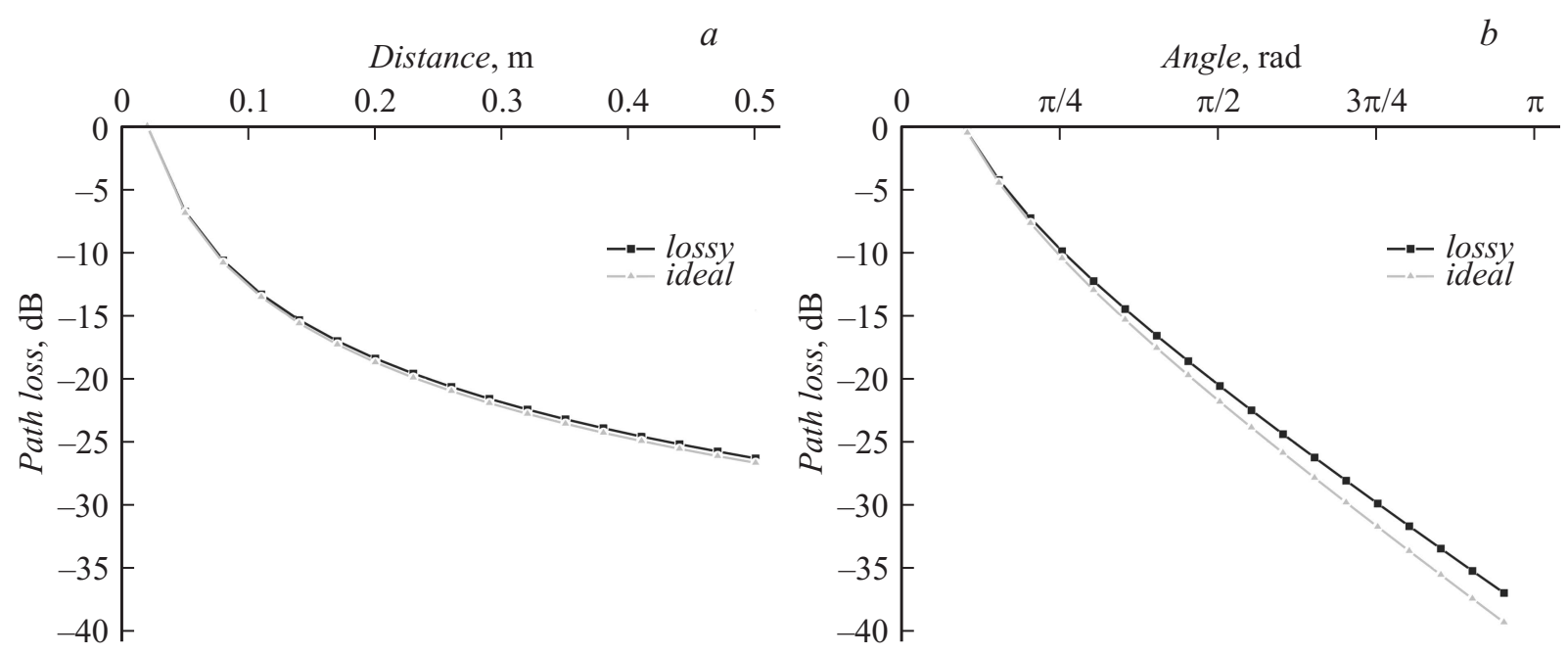

Рис. 5. Потери распространения ЭМ волны по плоской и цилиндрической поверхностям фантома: плоская поверхность $(a)$, цилиндрическая поверхность $(b)$.

Граничные условия для собственных функций этого уравнения заданы на границе диэлектрической поверхности кругового цилиндра и свободного пространства. Корни уравнения (11) определяются выражением

$$
t_{s}=t_{s}^{0}-\frac{q}{t_{s}^{0}}-\frac{q^{2}}{2\left(t_{s}^{0}\right)^{3}}+\frac{q^{3}}{\frac{1}{3}\left(t_{s}^{0}\right)^{2}+\frac{1}{2}\left(t_{s}^{0}\right)^{5}} \ldots
$$

где величина $t_{s}^{0}$ определена как

$$
t_{s}^{0}=\left(\frac{3 \pi}{2}\left(s-\frac{3}{4}\right)\right)^{2 / 3} e^{-\frac{i \pi}{3}}
$$

С использованием приведенных выражений (3)-(16) рассчитаем затухание ЭМ волны, распространяющейся вдоль плоского участка поверхности человеческого тела (фантома), представляющего собой однородный диэлектрик с проницаемостью $\varepsilon=42.5$ и удельной проводимостью $\sigma=1.66 \mathrm{~S} / \mathrm{m}$. Примем, что приемная и передающая антенны располагаются над поверхностью тела на расстоянии $5 \mathrm{~mm}$. На рис. $5, a$ показаны потери распространения ЭМ волны по плоской поверхности человеческого тела на частоте ЭМ волны $2.45 \mathrm{GHz}$, нормированные к потерям на расстоянии $20 \mathrm{~mm}$ от начала отсчета длины. На этом рисунке также приведены результаты расчета затухания ЭМ волны вдоль поверхности фантома, выполненного из идеального проводника. Расчет выполнялся с использованием выражения (3), в котором нормированный импеданс диэлектрической среды $\Delta_{0}$ принят равным нулю. Важно отметить, что разница в потерях распространения поверхностных волн по поверхности фантомов из диэлектрика с потерями и идеального проводника чрезвычайно мала. Природа потерь связана с существованием вытекающих волн, которые сосуществуют с поверхностной волной, прижатой к поверхности.
Зависимость потерь распространения ползущей волны от угла $\theta$ анализируется с использованием выражения (10). Для расчета приняты следующие параметры: радиус окружности $a=0.075 \mathrm{~m}$, расстояния от оси окружности до излучающей и приемной антенн одинаковые $b=r=0.080 \mathrm{~m}$, обе антенны располагаются над окружностью на расстоянии $5 \mathrm{~mm}$, частота ЭМ волны $2.45 \mathrm{GHz}$. Параметры диэлектрического слоя с потерями такие же, как и при расчете распространения ЭМ волны над плоской поверхностью. Расчет потерь распространения ползущей ЭМ волны для фантома из идеального проводника выполняется по формуле (10) при нулевом значении нормированного импеданса $\Delta_{0}$. На рис. 5, $b$ приведены потери распространения для фантома из неидеального диэлектрика и фантома из идеального проводника, нормированные к потерям для угла $\pi / 10$ или $24 \mathrm{~mm}$ вдоль длины окружности.

\section{4. Упрощенная модель распространения ЭМ волны вдоль поверхности}

Для выполнения оценочных расчетов параметров распространения ЭМ волны вдоль криволинейной поверхности человеческого тела найдем приближенную зависимость фазовой скорости ЭМ волны на круглой поверхности фантома, выполненного из идеального проводника, от радиуса этой поверхности. Выберем отрезок пути на поверхности цилиндра радиуса $R$, соответствующий радиальному углу $\Theta$ (рис. $6, a$ ). Дальнейший анализ выполним при следующих ограничениях, определяющих соотношения между характерными величинами, представленными на рис. $6, a$ :

$$
\lambda_{\text {eff }}<\lambda_{0} \ll R \text {. }
$$



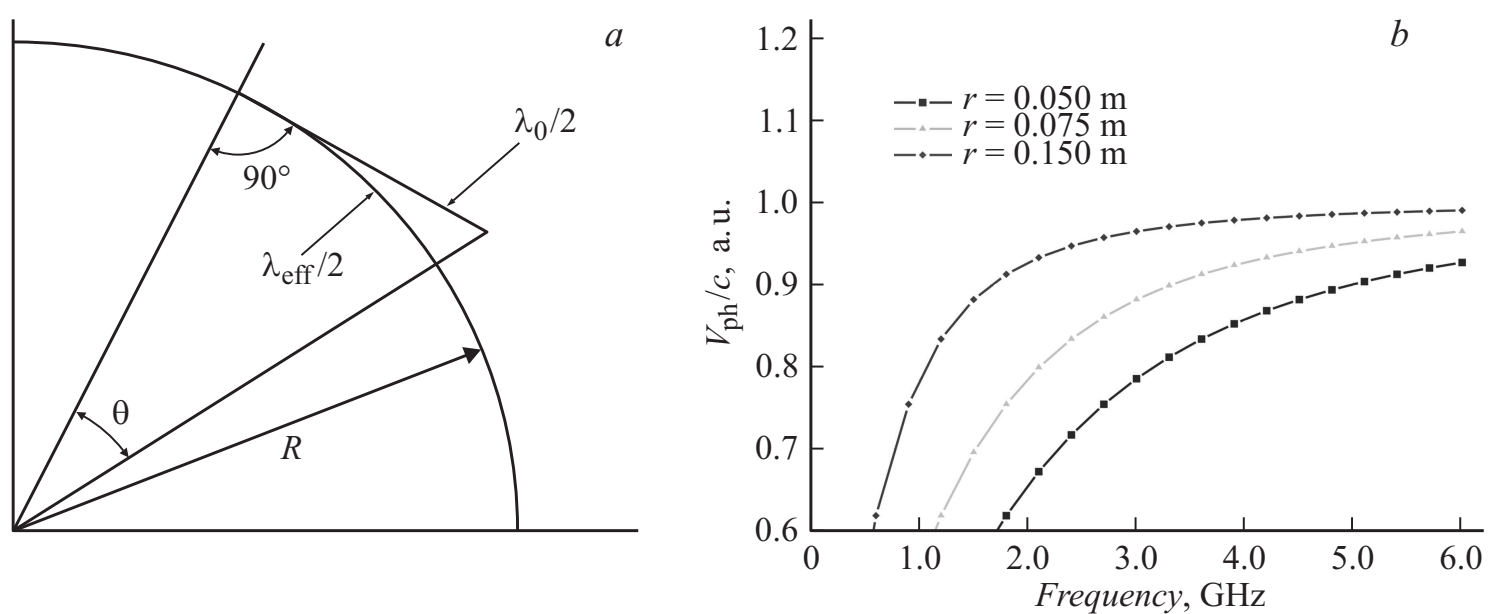

Рис. 6. Геометрическая схема для получения приближенной зависимости фазовой скорости ползущей ЭМ волны от радиуса криволинейной поверхности $(a)$. Результаты расчета частотной зависимости нормированной фазовой скорости $V_{\text {eff }} / c$ на цилиндрической поверхности $(b)$.

Выберем отрезок криволинейной поверхности, соответствующий углу $\Theta$, на котором укладывается половина

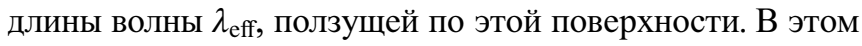
случае справедливо соотношение

$$
\frac{\lambda_{\mathrm{eff}}}{2}=\Theta R
$$

За то же время волна с длиной $\lambda_{0}$ в свободном пространстве распространяется по касательной к цилиндру и проходит путь $\lambda_{0} / 2$, соответствующий длине катета прямоугольного треугольника на рис. $6, a$ :

$$
\frac{\lambda_{0}}{2}=R \tan (\Theta) \text {. }
$$

Определим угол $\Theta$ из (19) и подставим найденное значение в (18). После преобразования найдем

$$
\lambda_{\text {eff }}=2 R \operatorname{arctg}\left(\frac{\lambda_{0}}{2 R}\right) .
$$

В выражении (20) перейдем от длины волны к фазовой скорости

$$
v_{\text {eff }}=2 f R \operatorname{arctg}\left(\frac{c}{2 f R}\right),
$$

где $c$ - фазовая скорость ЭМ волны на частоте $f$ в свободном пространстве и $v_{\text {eff }}-$ фазовая скорость ползущей волны на той же частоте.

На рис. 6, $b$ представлены зависимости нормированной фазовой скорости ползущей ЭМ волны $v_{\text {eff }} / c$ от частоты для различных радиусов цилиндрической поверхности круглого сечения.

Фазовая скорость ползущей ЭМ волны обладает дисперсией. Она увеличивается с ростом частоты, асимптотически приближаясь к скорости света в свободном пространстве в высокочастотном пределе. Качественно полученная зависимость весьма близка к данным, полученным в результате строгого решения задачи распространения ЭМ вдоль криволинейной поверхности.

\section{5. Экспериментальное исследование распространения ЭМ волны вдоль поверхности человеческого тела}

Экспериментальные исследования распространения ЭМ волн по поверхности человеческого тела проводились с целью верификации теоретических методов расчета параметров этих волн. Исследование выполнялось с использованием тела реального объекта (волонтера). В ходе экспериментов мощность СВЧ сигнала, излучаемого антенной, размещенной над поверхностью объекта, не превышали норм, установленных СанПиН [16].

В эксперименте исследовались потери распространения поверхностной и ползущей волн, а также дисперсионные характеристики каждого из двух типов волн. Две дисковые антенны (рис. 7,a), изготовленные с теми же размерами, что и антенна, используемая при моделировании структуры фантома, представленной на рис. 2, $a, b$, использовались в экспериментах в качестве источника и приемника ЭМ волн. Измерения потерь распространения проводились с использованием векторного анализатора цепей фирмы Rohde \& Schwarz ZVA8. На рис. 7, $b$ показана измеренная характеристика коэффициента отражения антенны.

Излучающая антенна во всех экспериментах располагалась на расстоянии $5 \mathrm{~mm}$ над поверхностью вблизи грудной мышцы с левой стороны тела (рис. 7,c, большая звездочка справа), приемная антенна перемещалась с постоянным шагом вдоль заранее заданной траектории также на расстоянии $5 \mathrm{~mm}$ над поверхностью тела. Поверхность тела размечалась метками с интервалом $50 \mathrm{~mm}$. Для исследования поверхностной волны траектория шла по вертикальной линии вниз до уровня пояса. Далее эта траектория называется „вертикальной“. Траектория, предназначенная для исследования ползу- 


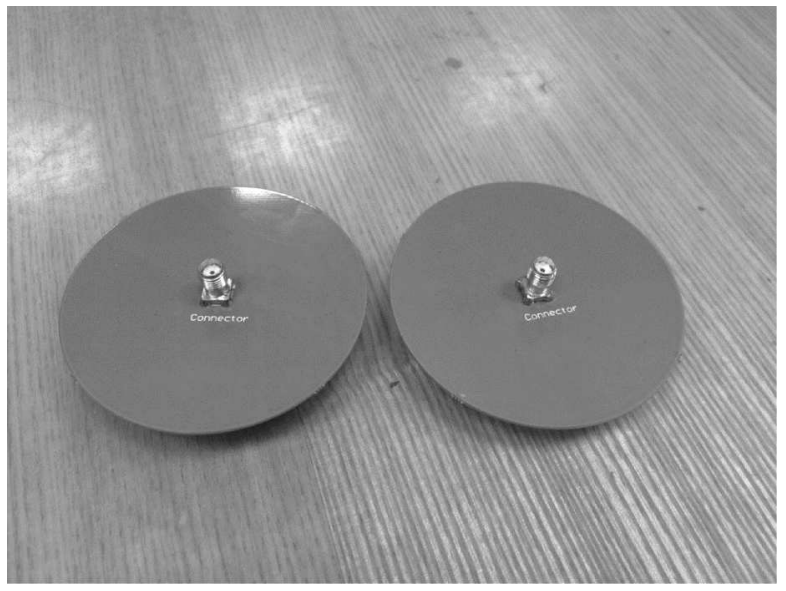

C

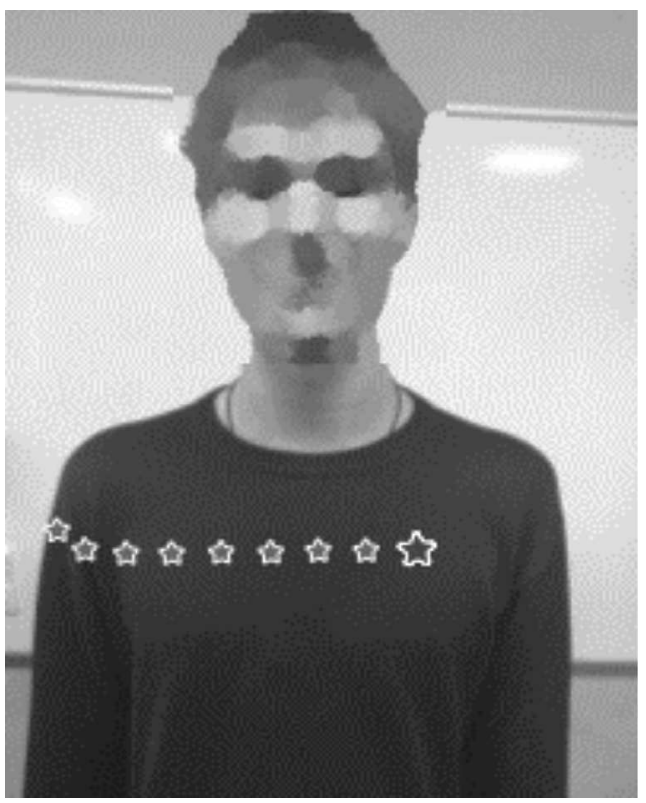

Frequency, GHz

$b$

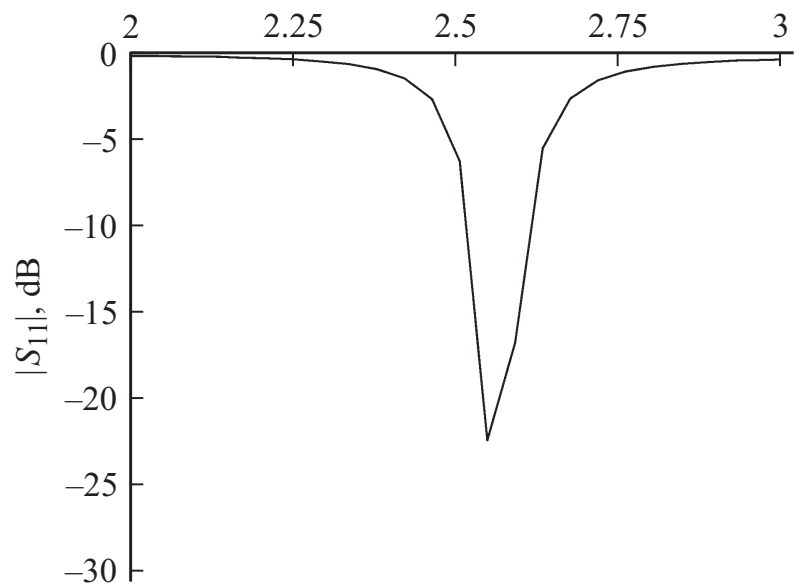

$d$

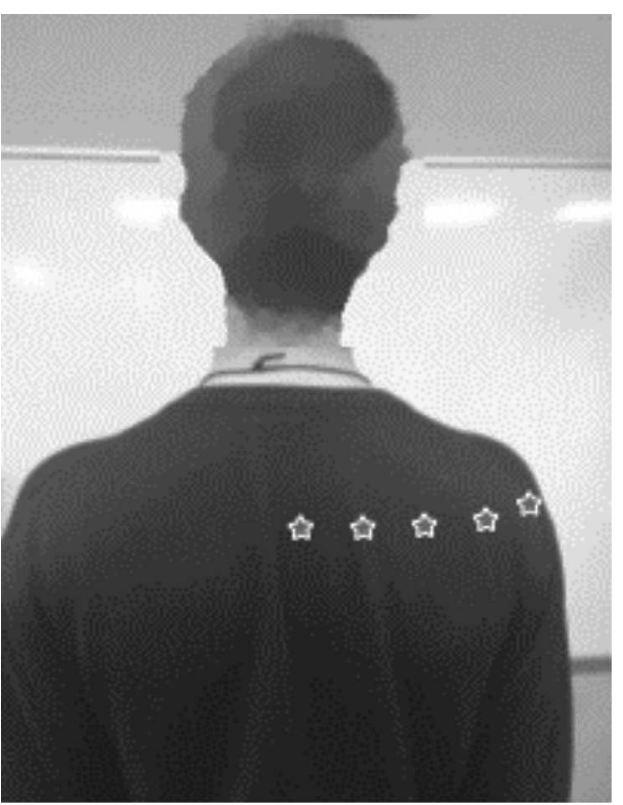

Рис. 7. Антенны, используемые для экспериментальных исследований $(a)$, и экспериментальная частотная зависимость коэффициента отражения на входе антенны $(b)$. Траектории, вдоль которых производились измерения потерь распространения: на груди $(c)$ и со спины $(d)$. Звездочкой справа отмечено положение излучающей антенны.

щей волны, шла от излучающей антенны параллельно уровню пола до середины спины (рис. 7,d) - эта траектория названа „горизонтальной“. Следует отметить, что ползущая волна существует только на отдельном участке „горизонтальной“ траектории в области плеча. Для волны, распространяющейся по „горизонтальной“ траектории, можно выделить три разных участка: первый отвечает распространению поверхностной волны вдоль грудной клетки и заканчивается на расстоянии $25 \mathrm{~mm}$ от излучающей антенны, второй, располагающийся на расстоянии от 25 до $35 \mathrm{~mm}$, принадлежит ползущей волне, огибающей плечо, и третий соответствует поверхностной волне, распространяющейся по горизонтали вдоль спины. Следует отметить замедление роста потерь распространения, начиная примерно с рассто- яния $45 \mathrm{~mm}$, что связано с передачей энергии между антеннами не только по „горизонтальной“ траектории, но и через область плеча. Потери распространения волны, распространяющейся по „вертикальной“ траектории, особенностей не имеют.

В другом эксперименте определялась длина ЭМ волны, распространяющейся по поверхности грудной клетки (поверхностная волна) и плечу (ползущая волна). Излучающая антенна располагалась так же, как и в первой части эксперимента, над левой грудной мышцей на расстоянии $5 \mathrm{~mm}$ от поверхности тела. Короткозамыкающая плоскость (тонкая пластина из жести прямоугольной формы площадью $150 \times 300 \mathrm{~mm})$ перемещалась вдоль поверхности в направлении удаления от антенны. Одновременно фиксировались положения 

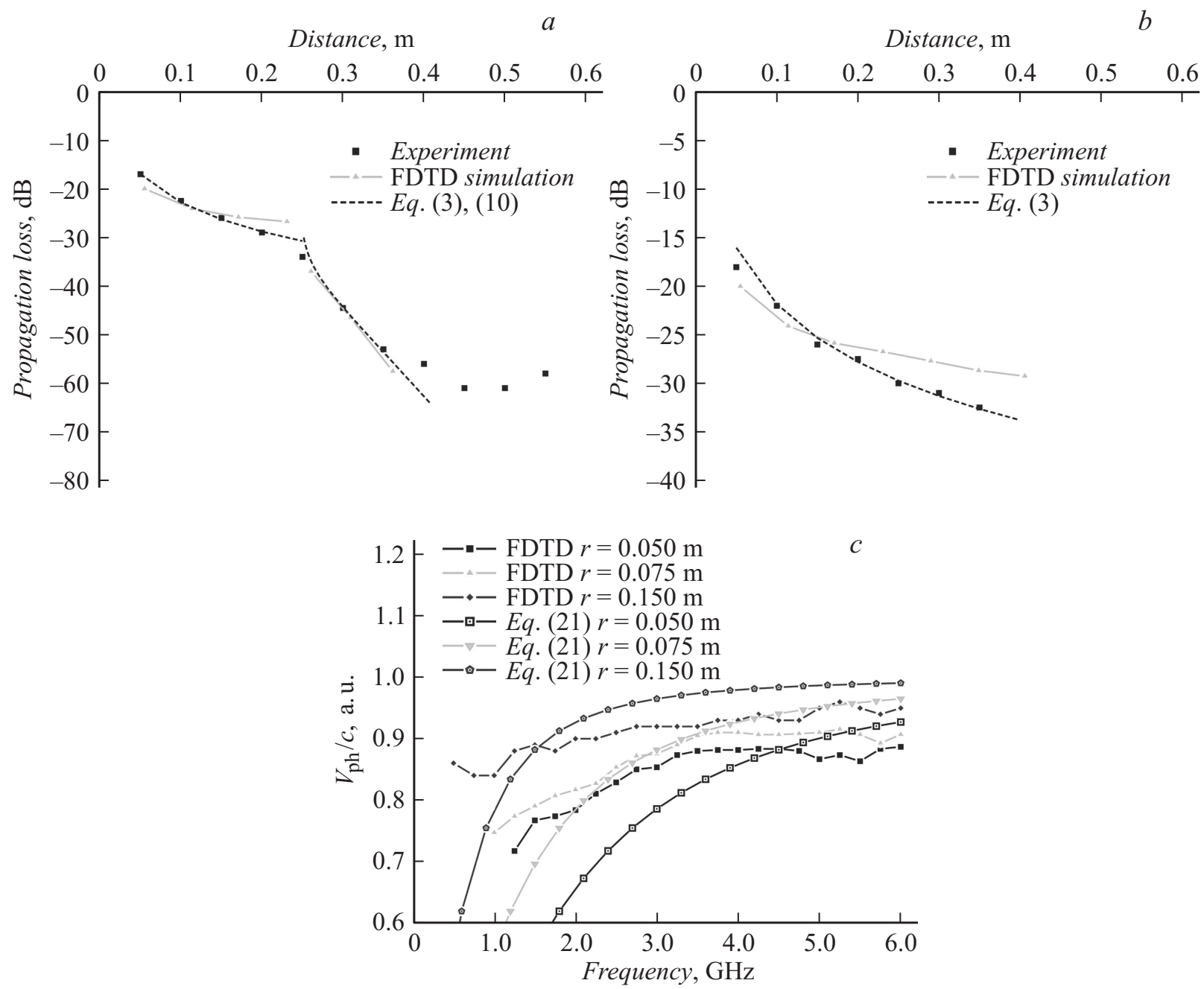

Рис. 8. Сравнение результатов эксперимента и расчета потерь распространения для „горизонтальной“ $(a)$ и „вертикальной“ $(b)$ траекторий. Точками обозначены результаты эксперимента, сплошными линиями - результаты численного расчета, штриховыми линиями - результаты аналитического моделирования. Сопоставление рассчитанных по приближенной формуле (21) и с применением численных методов зависимостей фазовой скорости ползущей волны от частоты для различных радиусов цилиндрической поверхности круглого сечения $(c)$.

пластины по удалению от антенны, при которых коэффициент отражения антенны на частоте ее излучения принимал максимальные значения. Расстояние $l$ между такими двумя положениями принималось равным половине длины волны, длина волны рассчитывалась по приведенной выше формуле (1). Для расчета фазовой скорость использовалось выражение (2).

Результаты эксперимента совместно с результатами расчетов, выполненных с использованием численных и аналитических методов, представлены в следующем разделе.

\section{6. Сравнение результатов и выводы}

Представленные в предыдущих разделах расчетные и экспериментальные данные позволяют провести сравнение численных и аналитических методов расчета потерь распространения ЭМ волн с результатами экспериментальных исследований и обосновать применимость упрощенных аналитических методов расчета.

Выберем геометрические параметры фантома, соответствующие реальному человеку, на поверхности тела которого проводились измерения потерь распространения. Данные геометрические параметры после соответствующих измерений сведены в табл. 1 .

На рис. 8, $a, b$ приведены экспериментальные зависимости потерь распространения для „горизонтальной и „вертикальной“ ${ }^{\circ}$ траекторий. На экспериментальные

Таблица 1. Размеры экспериментального объекта

\begin{tabular}{c|c|c|c|c}
\hline Параметр & Высота & Ширина & Толщина & Диаметр плеча \\
\hline Размер, mm & 1.785 & 0.420 & 0.250 & 0.105
\end{tabular}


Таблица 2. Сравнение характеристик ползущей и поверхностной ЭМ волн с частотой $2.55 \mathrm{GHz}$, полученных в результате численного моделирования, аналитического расчета и экспериментальных исследований

\begin{tabular}{l|c|c|c|c|c|c}
\hline & \multicolumn{2}{|c|}{$\begin{array}{c}\text { Численное } \\
\text { моделирование }\end{array}$} & \multicolumn{2}{|c}{$\begin{array}{c}\text { Аналитический } \\
\text { расчет (3)-(16) }\end{array}$} & \multicolumn{2}{|c}{ Эксперимент } \\
\cline { 2 - 7 } & ползущая & поверхностная & ползущая & поверхностная & ползущая & поверхностная \\
\cline { 2 - 7 } Длина волны, m & 0.102 & 0.116 & 0.089 & 0.122 & 0.097 & 0.114 \\
Нормированная фазовая скорость, $v_{\mathrm{eff}} / c$ & 0.867 & 0.986 & 0.730 & 1.000 & 0.825 & 0.969
\end{tabular}

графики на данных рисунках наложены зависимости, полученные в результате численного моделирования (разд. 2) и аналитического расчета (разд. 3) для геометрических параметров фантома, приведенных в табл. 1 . В качестве материала фантома в расчете выбран идеальный проводник.

На рис. 8, $a$, как уже было указано в разд. 5, присутствуют три участка, два из которых представляют собой плоские поверхности грудной клетки и спины, третий (в середине) - цилиндрическую поверхность, соответствующую плечу. Результаты аналитического моделирования, изображенные на рис. $8, a$, накладывались на экспериментальную кривую с вариацией начальной величины вносимых потерь для каждого из трех участков. На рис. $8, b$ присутствует единственная „вертикальная“ траектория. Начальные участки обеих траекторий представляют собой плоские поверхности, по которым распространяется поверхностная волна, и этот участок хорошо описывается моделью плоской волны. Следующий участок, присутствующий только на „го-

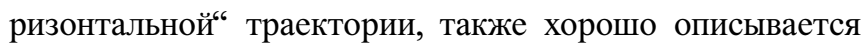
моделями. Третий участок „горизонтальной“ траектории заметно хуже представлен моделями. Особенность этого участка заключается в наличии сразу нескольких путей распространения ЭМ волн от излучающей антенны к приемной через область плеч.

Сравним также расчетные значения длины волны и фазовой скорости с результатами эксперимента. На рис. 8, с приведены результаты аналитического расчета по приближенной формуле (21) нормированной фазовой скорости ползущей волны в функции от частоты при разных диаметрах цилиндра, вдоль поверхности которого распространяется волна. Для сравнения на графике присутствуют точки, полученные в результате численного моделирования с применением метода FDTD (разд. 2). Приведенные результаты свидетельствуют о возможности выполнения оценочных расчетов параметров распространения ЭМ волны вдоль криволинейной поверхности.

В табл. 2 сведены результаты численного моделирования, аналитического расчета и экспериментальные данные для длины волны и фазовой скорости ЭМ волны, распространяющейся вдоль плоской и криволинейной поверхностей.
Результаты, представленные в табл. 2, показывают, что расчет фазовой скорости ползущей ЭМ волны аналитическим и численным методами достаточно хорошо коррелирует с экспериментальными результатами.

\section{Заключение}

Полученные результаты теоретического и экспериментального исследований распространения ЭМ волн по поверхности человеческого тела позволяют сделать следующие выводы:

- для моделирования распространения ЭМ волн СВЧ диапазона как численными, так и аналитическими методами можно использовать модель тела, выполненную из идеального проводника; точность выполняемых расчетов при этом достаточно высока и пригодна для практических приложений,

- аналитическое и численное моделирование методом FDTD позволяет достаточно точно рассчитать зависимость потерь распространения ЭМ волны от расстояния, пройденного волной, и типа поверхности (плоская или криволинейная),

- в работе выполнено исследование дисперсионных характеристик поверхностных и ползущих волн; выявленная дисперсия ползущей волны должна учитываться в практических приложениях.

Публикация выполнена в рамках государственной работы „Проведение научно-исследовательских работ (фундаментальных научных исследований, прикладных научных исследований и экспериментальных разработок)“ проектной части государственного задания Минобрнауки России (задание № 8.2579.2014/К).

\section{Список литературы}

[1] Balanis C.A. Antenna Theory: Analysis and Design, 3rd Edition. Wiley-Interscience, 2005.

[2] Collin R. Field Theory of Guided Waves. Wiley-IEEE Press, 1990.

[3] Persson P. Analysis and Design of Conformal Array Antennas, Ph.D. Thesis. Sweden: Univ. Serv. US AB, 2001.

[4] Paknys R., Jackson D.R. // IEEE Trans. on Antennas and Propag. 2005. Vol. 53. N 3. P. 898-907. 
[5] Conway G.A., Scanlon W.G., Cotton S.L., Bentum M.J. // URSI Intern. Symp. on Electromagnetic Theory. IEEE. 2010. P. 332-338.

[6] Hall P.S., Hao Y. Antennas and propagation for body-centric wireless communications. 2nd edition. Boston: Artech House, 2012. P. 54-58.

[7] Фок В.А. Проблемы дифракции и распространения электромагнитных волн. М.: Сов. радио, 1970.

[8] Хёнл Х., Мауэ А., Вестпбаль К. Теория дифракции. Пер. с нем. / Под ред. Г.Д. Малюжинца. М.: Мир, 1964.

[9] Wait J.R. // IRE Transactions on Antennas and Propagation. 1960. Vol. 8. N 4. P. 445-448.

[10] Pleskachev V., Vendik I., Vendik O., Kirillov V., Turalchuk P., Odit M. // Accepted for publ. EuCAP, 2016.

[11] Norton K.A. // Part I. Proc. Inst. Radio Eng. 1936. Vol. 24. N 10. P. 1367-1387.

[12] Norton K.A. // Part II. Proc. Inst. Radio Eng. 1937. Vol. 25. N 9. P. $1203-1236$.

[13] James R.W. Electromagnetic Waves in Stratified Media. Oxford University Press, 1996.

[14] Maclean J., Wu G. Radiowave Propagation Over Ground. Springer, 1993.

[15] Плескачев В.В., Вендик И.Б., Вендик О.Г. // Сб. трудов конференции „Электроника и микроэлектроника СВЧ“. СПб.: Изд-во ЛЭТИ, 2015. С. 202-206.

[16] Гигиенические требования к размещению и эксплуатации передающих радиотехнических объектов. СанПиН 2.1.8/2.2.4.1383-03. Федеральный центр Госсанэпиднадзора Минздрава России. 2003. Вып. 2003. 